\title{
Estimation of the effect of non-isotropically distributed
}

\section{energy on the apparent arrival time in correlations}

\author{
Bérénice Froment $^{1}$, Michel Campillo ${ }^{1}$, Philippe Roux ${ }^{1}$, Pierre Gouédard $^{2}$, Arie \\ Verdel $^{3}$ and Richard L. Weaver ${ }^{4}$ \\ ${ }^{1}$ Laboratoire de Géophysique Interne et de Tectonophysique, Université Joseph Fourier, \\ CNRS, Grenoble, France. E-mail: fromentb@obs.ujf-grenoble.fr; \\ michel.campillo@obs.ujf-grenoble.fr; philippe.roux@obs.ujf-grenoble.fr. \\ ${ }^{2}$ Department of Earth, Atmospheric and Planetary Sciences, Massachusetts Institute of \\ Technology, Massachusetts, USA.E-mail: gouedard@MIT.EDU. \\ ${ }^{3}$ Shell International Exploration and Production B.V., Rijswijk, The Netherlands. \\ E-mail: arie.verdel@shell.com. \\ ${ }^{4}$ Department of Physics, University of Illinois at Urbana-Champaign, Urbana, Illinois, \\ USA.E-mail: r-weaver@uiuc.edu.
}

(March 4, 2010)

Running head: Correlation of non-isotropic fields

\begin{abstract}
Correlations of random seismic noise are now widely used to retrieve the Green's function between two points. Whereas this technique provides useful results in tomography and monitoring studies, it is mainly limited by an uneven distribution of noise sources. In that case, theoretical requirements are not completely fulfilled and we may wonder how reliable the signals are reconstructed, in particular for the purpose of estimating traveltime from correlations. We present in this study a way to quantify effects of a non-isotropic noise
\end{abstract}


field by estimating the arrival time error due to a particular non-isotropic distribution of recorded wave intensity. Our study is based on a theoretical prediction of this bias and we successfully test the theory by comparing the theoretical expectation to real measurements from seismic-prospecting data. In particular, we distinguish between the effects of source distribution and effects of medium heterogeneity between the sources and the region of receivers. We find relative errors in the order of a percent which may affect monitoring results especially where smaller relative velocity variations (smaller than $10^{-3}$ for some applications) are investigated. Finally we see that correlation of coda waves helps mitigate the effects of a non-isotropic field, hence making the estimation of traveltime quite stable irrespective of the source distribution. 


\section{INTRODUCTION}

The Green's function of a medium between two points A and B represents the signal recorded at B if we would apply an impulse source at A: therefore the Green's function between any two given points $\mathrm{A}$ and $\mathrm{B}$ corresponds to the earth response between them. It has been demonstrated that with the crosscorrelation of random seismic wavefields, it is possible to retrieve the Green's function between two points.

Two acoustic experiments have initially demonstrated the possibility to reconstruct the Green's function by correlating diffuse wavefields (Weaver and Lobkis, 2001; Lobkis and Weaver, 2001). Afterwards, different theoretical approaches have formalised the link between correlation of random diffuse fields and Green's function retrieval (Weaver and Lobkis, 2001; Lobkis and Weaver, 2001; Derode et al., 2003a,b; van Tiggelen, 2003; Wapenaar, 2004; Snieder, 2004; Roux et al., 2005). The crosscorrelation technique has been used successfully in many applications in helioseismology (Duvall et al., 1993; Gilles et al., 1997), acoustics (Weaver and Lobkis, 2001; Lobkis and Weaver, 2001; Larose et al., 2004), oceanography (Roux and Kuperman, 2004; Sabra et al., 2005c), active seismic experiments (Bakulin and Calvert, 2006; Mehta et al., 2007) and seismology with the correlation of seismic coda (Campillo and Paul, 2003) or seismic noise (Shapiro and Campillo, 2004).

In seismology, it has been demonstrated that the correlation converges towards the Green's function as long as the different components of the elastic field follow the equipartition condition (Sanchez-Sesma et al., 2006, 2008). Practically, this corresponds to a selfaveraging process provided by randomization of the noise sources when considering long time series as well as scattering (Campillo, 2006; Larose et al., 2006; Gouédard et al., 2008b).

Even though correlations of seismic noise have provided useful information in tomo- 
graphic imaging (Shapiro et al. (2005); Sabra et al. (2005a) and recently Draganov et al. (2009) in which body wave reflections are retrieved) and monitoring studies (Brenguier et al., 2008a,b; Sens-Schonfelder and Wegler, 2006; Wegler and Sens-Schonfelder, 2007), the seismic noise is usually not perfectly diffuse and the conditions of the theory are not completely fulfilled. In that case, we may wonder how reliable the signals are reconstructed by the correlation especially for the purpose of estimating traveltimes: how does a non-isotropic distribution of sources affect the traveltime estimated from these noise correlations ?

Tomographic studies based on noise correlations use the traveltime of reconstructed Rayleigh waves to compute surface wave dispersion curves. Therefore, errors in traveltime lead to errors in tomographic results. Concerning monitoring studies, the evolution of traveltimes gives information about changes in the medium. But a change of noise sources may imply a change in traveltimes estimated from correlations which is not representative to a physical variation of the medium. Recent studies based on noise-based monitoring, have detected relative temporal changes of velocity smaller than $10^{-3}$ (Brenguier et al., 2008a,b) and further work is done to improve the accuracy of noise-based measurements. Considering such tiny variations, the influence of source distribution might be significant and should be quantified to correct it from measurements.

Previous studies have examined bias caused by non-isotropic source distribution in correlations. In an exploration application, van der Neut and Bakulin (2009) studied the amplitude distortions in correlations caused by an imperfect source illumination and proposed a method to correct results from this bias. Besides, Tsai (2009) and Yao and van der Hilst (2009) have shown that an uneven distribution of source intensity and medium heterogeneities can produce traveltime bias in noise-based measurements. From a tomographic point of view, Yao and van der Hilst (2009) develop an iterative procedure to correct tomo- 
graphic results for this bias. We adopt a somewhat different approach as we consider the forward problem and focus on the study of a direct formulation of this bias. In the next section, we illustrate the variability of apparent traveltime in correlations through results of a passive seismic noise study in the Parkfield area, California. Thereafter we present a theoretical expression for the error in arrival time due to a particular non-isotropic distribution of noise sources. In the last section, we compare the theoretical prediction to the real measurements on seismic prospecting data. In particular, we examine the effects of wave propagation in a heterogeneous medium surrounding the receivers and we discuss the advantages to correlate coda waves to mitigate effects of non-isotropy.

\section{ON THE STABILITY OF APPARENT TRAVELTIME IN NOISE CORRELATIONS AT THE SAN ANDREAS FAULT, PARKFIELD}

Between July 2001 and October 2002, 30 broadband stations located in an 11-km square, recorded continuous seismic noise in the Parkfield area, California. Plane wave beamforming is performed in $[0.1-0.2 \mathrm{~Hz}]$ to determine the direction of the seismic noise received by the Parkfield network. Figure 1 presents results of this beamforming analysis done on the entire network. In particular Figure 1(b) shows that the noise field is very directive, with a direction of about $55^{\circ}$ (North is $0^{\circ}$, positive rotation is clockwise). This direction is consistent with a noise origin in the Pacific Ocean (Sabra et al., 2005b; Stehly et al., 2006). Figure 1(a) shows the evolution of the beamformer outputs for 1-h data segments over 31 consecutive Julian Days (JD). This figure indicates daily fluctuations confirming that the seismic noise direction is highly time-dependent.

Consider two particular days (JD18 and JD22) for which the difference in noise direction 
is about $20^{\circ}$ (see Figure 1(a)): how does this azimuthal difference affect the traveltime in noise correlations ? We compute the 1-day averaged noise correlation function for two receiver pairs. The first pair (stations 8-27) is mostly aligned with the noise direction. By contrast, the second pair orientation (stations 13-30) is nearly orthogonal to the direction of energy flux (see Figure 2(a)). Figures 2(b) and 2(c) show results of the two dailycorrelations of interest (JD18 and JD22) for both station pairs. Whereas the time shift due to the variation in noise direction is negligible for pair 8-27, the effect on the $13-30$ receiver pair is significant since there is a relative time shift of about $30 \%$ for this case of extreme directive noise.

This particular extreme example shows that variability of apparent traveltime in correlations due to changes of a non-isotropic noise field may be significant. In order to get correct traveltime values, we must be able to quantify the effects of non-isotropy on traveltime estimates.

\section{THEORETICAL ERROR ON APPARENT TRAVELTIME DUE TO A NON ISOTROPIC DISTRIBUTION OF INTENSITY}

Weaver et al. (2009) derived a theoretical expression of the error in apparent arrival time in correlations due to a non-isotropic specific distribution of intensity. They consider (i) sources in far-field, (ii) the ballistic case and (iii) a homogeneous medium. The error is then evaluated in an asymptotic limit of large receiver-receiver separation. Results presented in this section correspond to this work.

Weaver et al. (2009) first show that in a fully asymptotic limit in which the two receivers are separated by a distance long compared to a wavelength $(x / \lambda \longrightarrow \infty)$, non-isotropy does 
not impair estimation of traveltime. However they go further by investigating the effects of smooth but non-isotropic intensity on traveltime estimates, at finite receiver separation (away from the full asymptotic limit).

We select two receivers, at positions $-x / 2$ and $x / 2$ around the origin (see Figure 3 ). We consider incoherent impulsive sources over an annular region, at large distance from the receivers. Since attention is confined to the two-dimensional case the field at the receivers may be viewed as a superposition of plane waves.

We consider the case that the recorded wavefield intensity distribution corresponds to the source intensity distribution which is controlled by an angular source weighting $B(\theta)(\theta$ is defined relative to the strike line connecting the receivers, (see Figure 3)). We consider smooth distributions by writing $B$ as a Fourier series:

$$
B(\theta)=B_{0}+B_{1} \cos (\theta)+B_{2} \cos (2 \theta)+B_{3} \cos (3 \theta)+\ldots
$$

Only cosines are used, by symmetry, as the receiver correlation does not distinguish between positive and negative $\theta$. This means that any distribution may be written as a symmetric distribution.

Considering that the time derivative of field correlation between both receivers $\left(C_{x}^{\prime}=\right.$ $\left.\frac{d}{d t} C_{x}(t)\right)$ converges to the Green's function (Gouédard et al., 2008b), $C_{x}^{\prime}$ gives an estimate of the traveltime $t$ between the two points. The predicted error in this apparent traveltime, due to non-isotropy is then (Weaver et al., 2009):

$$
\delta t \sim \frac{B^{\prime \prime}(0)}{2 t \omega_{0}^{2} B(0)} \quad \text { for positive correlation time }
$$




$$
\delta t \sim \frac{B^{\prime \prime}(180)}{2 t \omega_{0}^{2} B(180)} \quad \text { for negative correlation time }
$$

with $\omega_{0}$ the central angular frequency of the correlation waveform and angles expressed in degree. A positive error means an apparent arrival time earlier than the true arrival time by an amount of $\delta t$.

Equation 2 suggests that the error in apparent traveltime due to a non-isotropic distribution of intensity $B(\theta)$ decreases for high frequency and large receiver separation which is consistent with results by Tsai (2009) and Yao and van der Hilst (2009). Furthermore, we note that this expression gives an error proportional to the second derivative of the intensity $B$ evaluated in the receiver pair direction $\left(\theta=0^{\circ}\right.$ for positive correlation time). The stationary phase theorem helps in interpreting the physics aspect of this observation. Snieder (2004), Roux et al. (2005) explain the reconstruction of the Green's function by correlation techniques in the case of a homogeneous body, by using the stationary phase theorem: only sources in the alignment of the receivers contribute to reconstruction. End-fire lobes represent areas where those coherent sources are located. The aperture of this zone depends on the square root of the ratio between wavelength and distance between receivers: the higher the frequency, the narrower the end-fire lobes (Roux and Kuperman, 2004; Larose, 2005; Spetzler and Snieder, 2004). In the asymptotic limit considered, coherent reconstruction is then controlled by $\theta$ approaching 0 (for positive correlation time). But as we have seen before, we consider the case away from the full asymptotic limit. In that case the intensity near $\theta=0^{\circ}$ may be written as $B(\theta)_{\theta \rightarrow 0}=B(0)+\frac{1}{2} B^{\prime \prime}(0) \theta^{2}$ introducing a higher order term in the problem (the symmetry leads to $B^{\prime}(0)=0$ ). Since this term illustrates the difference with the full asymptotic case (and no error), it makes sense that it controls the traveltime 
error. For simplicity, we have only discussed the positive correlation time, but the argument is the same for negative time.

\section{ESTIMATION OF THE TRAVELTIME ERROR ON SEISMIC-PROSPECTING DATA}

\section{Acquisition geometry and data selection}

Petroleum Development Oman (PDO) recorded a high-resolution survey in northern Oman. The acquisition grid consists of 160025 -m-spaced receivers covering a $1 \times 1-\mathrm{km}$ area. The sources (vibrators) are located on a similar square grid shifted with respect to the receiver grid by $12.5 \mathrm{~m}$ in both directions. The complete data set then consists of $1600 \times 1600$ time-domain signals.

For this study, two receivers at the center of the array are selected. They are separated by a distance of $155 \mathrm{~m}$, corresponding to a surface wave traveltime of about $0.13 \mathrm{~s}$ at 15 $\mathrm{Hz}$ (wavelength $\sim 80 \mathrm{~m}$ ). We consider 240 sources in a 300-m-inner-radius, 70-m-thick ring centered on the receivers (see Figure 4(a)).

\section{Estimation of the error with respect to an isotropic distribution of sources}

In order to test the theoretical expression derived by Weaver et al. (2009), we compare the above predictions with measurements on these experimental data. Real measurements consist in estimating the time shift in the non-isotropic case with respect to a reference traveltime. In this section, the reference traveltime stems from the reference waveform obtained for an isotropic distribution of sources around receivers $(B(\theta)=$ constant $)$. 
Signals from the 240 sources are $(i)$ filtered between 10 and $20 \mathrm{~Hz},(i i)$ windowed into the range 0 to 1.5 seconds to emphasize ballistic waves (see Figure 4(b)) and (iii) correlated between both receivers. Results are then summed over sources with an angular weighting $B(\theta)$. The waveform obtained is thus the correlation between receivers of interest for the specific source distribution $B(\theta)$. Time shift $\delta t$ (that is, the traveltime error) due to non-isotropy of source distribution is then obtained by cross correlating the arrivals as constructed with $B(\theta)=$ constant and $B(\theta)$ of interest. To give relative time shifts, we estimate the arrival time $t$ by the maximum of the reference wavelet envelope. Finally, to enhance the quality of results, we azimuthally average these measurements. We thus consider 17 other pairs of receivers in the same region, oriented in two main directions (about $-130^{\circ}$ and $\left.-40^{\circ}\right)$ and spaced by distance between 150 and $158 \mathrm{~m}(\theta$ is redefined for the different pair orientations). Theoretical predictions result from equation 2 using a frequency of $15 \mathrm{~Hz}$ and a traveltime of $0.13 \mathrm{~s}$.

Results obtained for two different kinds of distribution (see Figures 5(a) and 5(c)) are presented in Figure 5. We can see that theoretical predictions fit the data measurements well for both examples. The asymptotic theory seems to describe successfully the apparent time shifts in real data, provided an intensity distribution sufficiently smooth (precise quantitative assessment of "sufficient smooth" is an asymptotic issue and has not yet been studied) and despite (i) simplifying assumptions behind equation 2 (plane waves in homogeneous medium) and (ii) receiver separation as small as two wavelengths. It is worth noting that the case presented in Figure 2(c) for the Parkfield region, may be explained by this theory considering a steep distribution of the form $B(\theta)=1+B_{2} \cos (2 \theta)$ shown in Figure 5(c). In that case, the theoretical time shift obtained with equation 2 using values involved in the Parkfield case (we take $t=3 \mathrm{~s}$ and $\omega_{0}=2 \pi \times 0.15=0.94 \mathrm{rad} / \mathrm{s}$ ) and $B_{2}=-0.6$ 
corresponds to the very large time shift observed (about 30\%).

However, these results are based on the hypothesis that an isotropic distribution of sources correspond to a perfect isotropic illumination. But the medium between the sources and the region of receivers is heterogeneous (Gouédard, 2008) and some propagation effects may modify the wavefield making the effective intensity distribution in the vicinity of receivers non-isotropic despite the isotropic source distribution. In that case, the reference taken in these results does not correspond to the actual isotropic case and the previous estimated time shift is not the over-all error.

\section{Effective distribution of intensity for an isotropic distribution of sources}

The goal of this section is to determine the effective intensity distribution received at receivers for an isotropic distribution of sources. By doing so, we will be able to highlight and quantify possible propagation effects on traveltime estimates. The analysis is done under assumption of homogeneity between the receivers.

Plane-wave beamforming is performed to get directional features of the wavefield. We use 37 stations of the network centered on the same position as the center of the receiver positions considered before, and the same 240 sources as used previously. The geometry is shown in Figure 7(a). Beamforming is computed for each of the 240 different sources on the entire sub-array formed by the 37 receivers. The total distribution may then be seen as the sum of all source contributions. Processing consists in considering [0-1.5 s] whitened signals (i.e., the temporal window corresponding to the ballistic waves) and summing incoherently beamformer outputs over 6 frequencies between 10 and $15 \mathrm{~Hz}$.

Figure 6(a) shows an example of beamformer output for a single source whose location 
is indicated in Figure 7(a). In order to highlight some propagation effects, we compare the angular position of the source with the angular direction of incoming energy. The difference observed indicates that energy has been deflected during the propagation into the medium between the sources and the region of receivers (about $10^{\circ}$ for this particular source). This change in direction is due to velocity variations in the medium. Such deflections $\left(10^{\circ}\right)$ may be caused by realistic relative velocity heterogeneities of about $10 \%$ (see Figure $7(\mathrm{a})$ ). The deflection is different for the different sources (see Figure 6(b)) suggesting complicated wavefields at receivers.

Assuming that the sum of beamforming results over sources represents the actual energy pattern at the center of the network, we can define the effective intensity distribution at receivers as the total of maximum energy at every angle (see Figure 7 (b) for an isotropic distribution of sources). To get closer to theory conditions, we smooth the distribution by averaging intensity in a sliding $40^{\circ}$-wide angular window $\left(40^{\circ}\right.$ corresponding to the size of the end-fire lobe for the frequency considered).

These results show that isotropic distribution of sources does not necessarily lead to isotropic distribution of intensity and thus does not correspond to the ideal isotropic illumination. These observations mean that intensity distribution is partly controlled by wave propagation in the heterogeneous medium surrounding the receiver region. The next section is devoted to quantify the propagation effects on traveltime estimates to take them into account in the determination of a total error. 


\section{Incorporation of propagation effects in the estimation of the error in trav-} eltime

The previous section has allowed us to determine the contribution of propagation to the intensity distribution. For the sake of simplicity, this contribution (displayed in Figure 7(b)) will be called $B_{p}(\theta)$.

In order to quantify the relation between propagation effects and traveltime estimates, we apply the theoretical expression given by equation 2 with $B_{p}(\theta)$. Note again that our analysis is done under the assumption of a local homogeneity of the medium in the vicinity of the paths between the receivers. As $B_{p}(\theta)$ varies azimuthally, effects on apparent traveltimes will depend on the angular orientation of receiver pairs. We found that relative time shifts may reach $1 \%$ for some particular receiver pair orientations (the larger errors are observed for directions of lower intensity in Figure 7(b)). In other words, raypath deflections due to medium heterogeneities may cause an error in the traveltime estimate that is not negligible with respect to errors due to a non-isotropic source distribution as seen in Figure 5. From the estimate of the part of the error due to propagation, how could we estimate the cumulative effect of medium heterogeneity and non-isotropy of sources on apparent traveltime?

We present here a simple analysis leading to an expression of the total bias. Consider a non-isotropic source distribution $\left(B_{s}(\theta) \neq\right.$ constant $)$; the actual intensity distribution may be written in first approximation as:

$$
B(\theta)=B_{s}(\theta) \times B_{p}(\theta)
$$

leading to an error of the form

$$
\delta t=\delta t_{s}+\delta t_{p} .
$$


Intermediate steps are detailed in Appendix A. A prediction of the actual error in traveltime due to non-isotropy could thus be obtained by summing the predicted time shift due to $(i)$ non-isotropy in source distribution, $\delta t_{s}$ (estimated in Figure 5) and (ii) propagation effects, $\delta t_{p}$

In order to control the reliability of our analysis, we would like to compare this bias estimate to reference traveltimes. The presence of active sources near receivers, allows us to directly acquire Green's functions between receivers of interest. These signals may thus be used to obtain reference measurements of traveltimes. The question is to know if the apparent traveltimes from correlations and corrected with $\delta t$ (given by equation 4) are consistent with this measurement. Since both arrays are shifted by $12.5 \mathrm{~m}$ in both directions, we use the closest sources to the receivers considered and we apply a correction on the time axis to correct for this distance difference. However, at the scale of precision considered here, direct measurements are much less stable than correlation measurements, making any comparison difficult. Different causes can explain the instability of direct estimates. First, the sources used in this case, are vibrators producing source signals with a 1-m-side plate. Considering receivers separated by about $150 \mathrm{~m}$, the spatial precision of the source location corresponds to the order of precision of the traveltime that we investigate (a few percent). Furthermore, receivers consist of clusters of 12 geophones adding possible imprecisions. Finally, some spectral aspects may make comparisons difficult between the active signal and the correlation function since the spectra of both signals are quite different and time estimates are strongly sensitive to spectral contents. 


\section{Mitigation of the effects of non-isotropy: correlation of coda waves}

Coda waves correspond to waves scattered from the heterogeneities in the earth (e.g., Aki and Chouet (1975)). Scattering tends to make the wavefield more isotropic depending on the distribution of the scatterers in the medium and the lapse time considered in the coda (Paul et al., 2005). Even if the field is not completely isotropic, the distribution of incoming energy should no longer be controlled exclusively by the source distribution but also by the scatterer distribution in the medium.

In order to study the influence of the source distribution when using coda waves, we investigate how time shifts on correlations of coda waves depend on the source distribution. We can note that in case of perfect isotropic coda wavefield, the expected bias will be zero $\left(B^{\prime \prime}(\theta)=0\right.$ in equation 2$)$. The idea is the same as with previous time shift measurements (see Figure 5), but instead of correlating direct waves, we select a later part of the signal corresponding to coda waves (see Figure (4(b)). The diffuse regime is indicated by the linear energy decay in decibel scale in this window: to cancel this decay, the decrease of recorded amplitudes in this time window is compensated. As previously, the reference waveform corresponds to the correlation function (here of coda waves) obtained for an isotropic distribution of sources and we compare time shift measurements to the ballistic theory.

However, the signal-to-noise ratio is much lower for correlation of coda waves (Gouédard et al., 2008a). In order to get accurate time measurements, we need to average correlations. Thus, in this section we measure time shifts on correlation functions averaged over the 18 receiver pairs of different orientations considered before.

Results presented in Figure 8 show that the time shift with respect to the reference is 
always about zero suggesting that arrival time does not highly depend anymore on source distribution. As in Figure 5, this result does not correspond to a total error, but even if this error is not zero (the scattered field may be still anisotropic) this time shift is almost independent of the distribution of sources. This can be related intuitively to equation 2 by considering that coda waves make the intensity distribution smoother (see Figures 6(a) and 6(c)). This leads to lower values of $B^{\prime \prime}(\theta)$ and thus smaller traveltime bias. This result confirms the interest of using coda waves in many applications involving uneven source distribution since Figure 8 shows that we mitigate the traveltime bias due to non-isotropic source distributions by a factor of 10 .

\section{CONCLUSION}

We have shown a way to estimate the error made in traveltime estimated from correlations due to non-isotropic recorded energy provided that the azimuthal distribution is sufficiently smooth.

Non-isotropy in source distribution is one of the main causes of non-isotropic energy. But in the case of a heterogeneous medium between sources and the region of receivers, propagation may modify the wavefield and make it more complicated, producing an additional error in traveltime. We have seen in this study that this effect is not negligible as compared to the effect of a non-isotropic source distribution. This means that in the case of a very heterogeneous medium, propagation effects should be taken into account in the error estimation. The method proposed in this paper provides a means to estimate the traveltime error taking into account both effects and allows us to predict errors smaller than $1 \%$.

We observe errors in the order of one percent. The influence of this error highly depends 
on applications: whereas this amount will not introduce misinterpretation in tomographic studies (recently confirmed by results from Yao and van der Hilst (2009)), it may affect strongly monitoring results since we investigate much smaller relative velocity variations

(smaller than $10^{-3}$ ). Therefore, this work highlights the interest to estimate the effect of non-isotropic distribution of energy to allow to correct noise-based measurements in monitoring studies for this effect.

Finally, we have seen that scattering may play a useful role in reducing this error since correlating coda waves instead of ballistic waves, allows us to mitigate strongly the influence of source distribution in traveltime estimates.

\section{ACKNOWLEDGMENTS}

The authors thank the Ministry of Oil and Gas of the Sultanate of Oman, Petroleum Development of Oman and Shell Research for permission to use the data. One of the authors (B.F.) acknowledges the support from Shell Research. This work is supported by the European Research Council (advanced grant Whisper).

\section{APPENDIX A}

\section{ACTUAL INTENSITY DISTRIBUTION FORMULATION AND ASSOCIATED TRAVELTIME ERROR}

The actual intensity distribution taking into account both source and propagation effects is written in the form

$$
B(\theta)=\int_{0}^{2 \pi} B_{p}^{*}\left(\theta^{\prime}, \theta\right) B_{s}\left(\theta^{\prime}\right) d \theta^{\prime}
$$


where $B_{p}^{*}\left(\theta^{\prime}, \theta\right)$ describes the contribution of unit sources located in $\theta^{\prime}$ to intensity in $\theta$.

In first approximation we write:

$$
B_{p}^{*}\left(\theta^{\prime}, \theta\right)=B_{p}(\theta) f\left(\theta-\theta^{\prime}\right)
$$

and we assume

$$
f\left(\theta-\theta^{\prime}\right) \sim \delta\left(\theta-\theta^{\prime}\right)
$$

By making this approximation, we neglect multipathing considering that all energy in the direction $\theta$, i.e. $B_{p}(\theta)$, comes from a single source located in $\theta$.

Equation A-1 then simplifies as:

$$
\begin{aligned}
B(\theta) & =\int_{0}^{2 \pi} B_{p}(\theta) \delta\left(\theta-\theta^{\prime}\right) B_{s}\left(\theta^{\prime}\right) d \theta^{\prime}, \\
B(\theta) & =B_{p}(\theta) \times B_{s}(\theta) .
\end{aligned}
$$

The approximation used here is particularly valid in moderately heterogeneous media or when sources are not too far from receivers (in the case considered, source-receiver separation is approximately $4 \lambda$ ). Such values make this approximation usable in longer period seismology, for example in the primary and secondary microseism band (5-15 s) and station distances in the order of 50-150 km (Shapiro et al., 2005).

From equation A-5, we derive:

$$
B^{\prime \prime}(\theta)=B_{s}^{\prime \prime}(\theta) \times B_{p}(\theta)+2 \times B_{s}^{\prime}(\theta) \times B_{p}^{\prime}(\theta)+B_{s}(\theta) \times B_{p}^{\prime \prime}(\theta)
$$

Since the correlation function does not distinguish between positive and negative $\theta$, we can write any distribution as a symmetric distribution. That's why we write $B_{s}$ in a Fourier series of $\cos (\theta)$ and for $\theta_{0}=0^{\circ}$ or $\theta_{0}=180^{\circ}, B_{s}^{\prime}\left(\theta_{0}\right)=0$. 
Then,

$$
\begin{aligned}
B^{\prime \prime}\left(\theta_{0}\right) & =B_{s}^{\prime \prime}\left(\theta_{0}\right) \times B_{p}\left(\theta_{0}\right)+B_{s}\left(\theta_{0}\right) \times B_{p}^{\prime \prime}\left(\theta_{0}\right), \\
\frac{B^{\prime \prime}\left(\theta_{0}\right)}{B\left(\theta_{0}\right)} & =\frac{B_{s}^{\prime \prime}\left(\theta_{0}\right)}{B_{s}\left(\theta_{0}\right)}+\frac{B_{p}^{\prime \prime}\left(\theta_{0}\right)}{B_{p}\left(\theta_{0}\right)}
\end{aligned}
$$

and from equation 2 :

$$
\delta t=\delta t_{s}+\delta t_{p}
$$




\section{REFERENCES}

Aki, K., and B. Chouet, 1975, Origin of coda waves: source, attenuation and scattering effects: Journal of Geophysical Research, 80, 3322-3342.

Bakulin, A., and R. Calvert, 2006, The virtual source method: theory and case study: Geophysics, 71, no. 4, SI139-SI150.

Brenguier, F., M. Campillo, C. Hadziioannou, N. Shapiro, R. Nadeau, and E. Larose, 2008a, Postseismic relaxation along the San Andreas fault at Parkfield from continuous seismological observations: Science, 321, 1478-1481.

Brenguier, F., N. Shapiro, M. Campillo, V. Ferrazzini, Z. Duputel, O. Coutant, and A. Nercessian, 2008b, Towards forecasting volcanic eruptions using seismic noise: Nature Geoscience, 1, 126-130.

Campillo, M., 2006, Phase and correlation in 'random' seismic fields and the reconstruction of the Green function: Pure and Applied Geophysics, 163, 475-502.

Campillo, M., and A. Paul, 2003, Long-range correlations in the diffuse seismic coda: Science, 299, 547-549.

Derode, A., E. Larose, M. Campillo, and M. Fink, 2003a, How to estimate the Green's function of a heterogeneous medium between two passive sensors? Application to acoustic waves: Applied Physics Letters, 83, 3054-3056.

Derode, A., E. Larose, M. Tanter, J. de Rosny, A. Tourin, M. Campillo, and M. Fink, 2003b, Recovering the Green's function from field-field correlations in an open scattering medium (L): Journal of the Acoustical Society of America, 113, 2973-2976.

Draganov, D., X. Campman, J. Thorbecke, A. Verdel, and K. Wapenaar, 2009, Reflection images from ambient seismic noise: Geophysics, 74, no. 5, A63-A67.

Duvall, T., S. Jefferies, J. Harvey, and M. Pomerantz, 1993, Time distance helioseismology: 
Nature, 362, 430-432.

Gilles, P., T. Duvall, P. Scherrer, and R. Bogart, 1997, A subsurface flow of material from the sun's equator to its poles: Nature, 390, 52-54.

Gouédard, P., 2008, Techniques de corrélation : aspects méthodologiques et applications à la sub-surface: PhD thesis, Université Joseph Fourier.

Gouédard, P., P. Roux, M. Campillo, and A. Verdel, 2008a, Convergence of the two-point correlation function toward the Green's function in the context of a seismic prospecting dataset: Geophysics, 73, no. 6, V47-V53.

Gouédard, P., L. Stehly, F. Brenguier, M. Campillo, Y. Colin de Verdière, E. Larose, L. Margerin, P. Roux, F. Sanchez-Sesma, N. Shapiro, and R. Weaver, 2008b, Crosscorrelation of random fields: Mathematical approach and applications: Geophysical Prospecting, 56, 375-393.

Larose, E., 2005, Diffusion multiple des ondes sismiques et expériences analogiques en ultrasons: PhD thesis, Université Joseph Fourier.

Larose, E., A. Derode, M. Campillo, and M. Fink, 2004, Imaging from one-bit correlation of wide-band diffuse wavefield: Journal of Applied Physics, 95, 8393-8399.

Larose, E., L. Margerin, A. Derode, B. van Tiggelen, M. Campillo, N. Shapiro, A. Paul, L. Stehly, and M. Tanter, 2006, Correlation of random wavefields: an interdisciplinary review: Geophysics, 71, no. 4, SI11-SI21.

Lobkis, O., and R. Weaver, 2001, On the emergence of the Green's function in the correlations of a diffuse field: Journal of the Acoustical Society of America, 110, 3011-3017.

Mehta, K., A. Bakulin, J. Sheiman, R. Calvert, and R. Snieder, 2007, Improving the virtual source method by wavefield separation: Geophysics, 72, no. 4, V79-V86.

Paul, A., M. Campillo, L. Margerin, E. Larose and A. Derode, 2005, Empirical synthesis 
of time-asymmetrical Green functions from the correlation of coda waves: Journal of Geophysical Research, 110, B08302.

Roux, P., and W. Kuperman, 2004, Extracting coherent wave fronts from acoustic ambient noise in the ocean: Journal of the Acoustical Society of America, 116, 1995-2003.

Roux, P., K. Sabra, W. Kuperman, and A. Roux, 2005, Ambient noise cross-correlation in free space: theoretical approach: Journal of the Acoustical Society of America, 117, $79-84$.

Sabra, K., P. Gerstoft, P. Roux, and W. Kuperman, 2005a, Surface wave tomography from microseisms in southern California: Geophysical Research Letters, 32, L14311.

Sabra, K., P. Gerstoft, P. Roux, W. Kuperman and M. Fehler, 2005b, Extracting timedomain Green's function estimates from ambient seismic noise: Geophysical Research Letters, 32, L03310.

Sabra, K., P. Roux, and W. Kuperman, 2005c, Arrival-time structure of the time-averaged ambient noise cross-correlation function in an oceanic waveguide: Journal of the Acoustical Society of America, 117, 164-174.

Sánchez-Sesma, F., J. Pérez-Ruiz, M. Campillo, and F. Luzón, 2006, Elastodynamic 2-D Green function retrieval from cross-correlation: canonical inclusion problem: Geophysical Research Letters, 33, L13305.

Sánchez-Sesma, F., J. Pérez-Ruiz, F. Luzón, M. Campillo, and A. Rodríguez-Castellanos, 2008, Diffuse fields in dynamic elasticity: Wave motion, 45, 641-654.

Sens-Schonfelder, C., and U. Wegler, 2006, Passive image interferometry and seasonal variations of seismic velocities at Merapi volcano, Indonesia: Geophysical Research Letters, 33, L21302.

Shapiro, N., and M. Campillo, 2004, Emergence of broadband Rayleigh waves from corre- 
lations of the ambient seismic noise: Geophysical Research Letters, 31, L07614.

Shapiro, N., M. Campillo, L. Stehly, and M. Ritzwoller, 2005, High-resolution surface-wave tomography from ambient seismic noise: Science, 307, 1615-1618.

Snieder, R., 2004, Extracting the Green's function from the correlation of coda waves: a derivation based on stationary phase: Physical Review E., 69, 046610.

Spetzler, J., and R. Snieder, 2004, The Fresnel volume and transmitted waves: Geophysics, 69, 653-663.

Stehly, L., M. Campillo, and N. Shapiro, 2006, A study of the seismic noise from its longrange correlation properties: Journal of Geophysical Research, 111, B10306.

Tsai, V., 2009, On establishing the accuracy of noise tomography travel-time measurements in a realistic medium: Geophysical Journal International, 178, 1555-1564.

van der Neut, J. and A. Bakulin, 2009, Estimating and correcting the amplitude radiation pattern of a virtual source: Geophysics, 74, no. 2, SI27-SI36.

van Tiggelen, B., 2003, Green function retrieval and time reversal in a disordered world: Physical Review Letters, 91, 243904.

Wapenaar, K., 2004, Retrieving the elastodynamic Green's function of an arbitrary inhomogeneous medium by crosscorrelation: Physical Review Letters, 93, 254301.

Weaver, R., B. Froment, and M. Campillo, 2009, On the correlation of non-isotropically distributed ballistic scalar diffuse waves: Journal of the Acoustical Society of America, 126, $1817-1826$.

Weaver, R., and O. Lobkis, 2001, Ultrasonics without a source: thermal fluctuation correlations at MHz frequencies: Physical Review Letters, 87, 134301.

Wegler, U., and C. Sens-Schonfelder, 2007, Fault zone monitoring with passive image interferometry: Geophysical Journal International, 168, 1029-1033. 
Yao, H., and R. van der Hilst, 2009, Analysis of ambient noise energy distribution and phase velocity bias in ambient noise tomography, with application to SE Tibet: Geophysical Journal International, 179, 1113-1132. 


\section{LIST OF FIGURES}

1 (a) Results of plane wave beamforming on the Parkfield network showing the variation of seismic noise direction between $0.1 \mathrm{~Hz}$ and $0.2 \mathrm{~Hz}$, for 31 consecutive days. Direction is represented by angle in degree (North is $0^{\circ}$, positive rotation is clockwise). The color scale corresponds to the maximum values of the beamformer, 1 corresponding to a perfect match between the data and the plane wave model. (b) Angular distribution of speed of incoming noise on Parkfield network for one day (North is $0^{\circ}$, positive rotation is clockwise). The color scale corresponds to the agreement between the data and the plane wave model (1 corresponding to a perfect match). The angular spot width matches the expected angular diffraction spot size of the network.

2 (a) Station map of the Parkfield Network. The black arrow shows the average seismic noise direction $\left(\sim 55^{\circ}\right)$ indicating that the noise is coming from the Pacific Ocean. Station pairs of interest are indicated by black lines. (b) and (c) 1-day averaged correlation function for two particular days, JD18 (gray line) and JD22 (black dashed line). Two different pairs are considered: the pair 8-27 (b) mostly aligned with the noise direction and, contrariwise, the pair 13-30 (c) perpendicular to the noise direction.

3 Geometry considered. Two receivers at the center of the network are separated by a distance $\mathrm{x}$. Sources are located in an annular region of large radius (compared to the wavelength of interest) centered on receivers. Each source $s$ is located by an angle $\theta$ defined relative to the line connecting the receivers (positive rotation is anti-clockwise).

4 (a) Experimental geometry. 240 sources are selected and located in an annulus of inner radius $300 \mathrm{~m}$ and thickness $70 \mathrm{~m}$, surrounding two receivers separated by $155 \mathrm{~m}$. (b) Example of intensity (decibel scale) at receivers averaged over the 240 sources selected. The thin black line represents the boundary between both temporal windows considered in this 
work: direct waves between 0 and $1.5 \mathrm{~s}$ and coda waves between 1.5 and $3 \mathrm{~s}$.

$5 \quad$ Measured (cross) and predicted (dashed line) relative time shifts for two different kinds of source distribution. Theoretical values result from equation 2. Error bars correspond to standard deviation of the 18 pair measurements. The first case corresponds to a distribution of the form $B(\theta)=1+B_{1} \cos (\theta)$. Figure (a) shows the shape of the distribution for $B_{1}=0$ (isotropic source distribution) and $B_{1}=-0.6$ for which most of the energy comes from the direction $\theta=180^{\circ}$. Different values of $B_{1}$ then correspond to different source intensity distribution. Results of relative time shifts are presented in (b) for $B_{1}$ evolving from 0 to -0.6 and for positive (above) and negative (below) correlation time. The reference traveltime corresponds to the correlation function obtained for an isotropic distribution of sources. A second case is presented in (c) and (d). The source distribution is of the form $B(\theta)=1+B_{2} \cos (2 \theta)$ where energy direction is mainly perpendicular to the receiver line. Relative errors on traveltimes reach $2 \%$, with a large variance, for the case of a steep distribution (example of $B_{2}=-0.6$ in case 2).

6 (a) Angular distribution of speed of incoming energy of ballistic waves, resulting from plane wave beamforming applied on a single source whose location is indicated in Figure 7(a). The black line corresponds to the angular position of the source. The angular coordinate system in (a), (b) and (c) is the same as the one displayed in Figure 7. The azimuthal difference between the source position and the direction of incoming energy shows a deflection in the medium during propagation between the sources and the region of receivers. Figure (b) presents values of the deflection versus the azimuthal position of sources, for the 240 sources considered. (c) Same as (a) except that we have considered the coda part of the signal (i.e., between 1.5 and $3 \mathrm{~s}$ ) in the beamforming analysis instead of ballistic waves. We can note that considering the coda part of the signal makes the intensity 
distribution smoother.

7 (a) Group-speed map of 12.5-Hz-period Rayleigh waves obtained from active data. Color represents the value of group velocity in m/s (from Gouédard (2008)). Gray dots and black triangles represent respectively the 240 sources and 37 receivers considered for the beamforming analysis. The black circle indicates the source corresponding to Figures 6(a) and 6(c). (b) Polar representation of the effective intensity distribution at receivers (gray line) for an isotropic distribution of sources (black dashed line); (a) and (b) have the same orientations.

8 Comparison between relative time shift measurements on coda waves correlations (cross) and expectation from ballistic theory given by equation 2 (dashed line) for positive (above) and negative (below) correlation time. We consider a source distribution of the form $B(\theta)=1+B_{2} \cos (2 \theta)$ (same as Figure $5(\mathrm{c})$ ). By contrast to Figure $5(\mathrm{~d})$, measurements are based on coda waves correlations. The reference traveltime corresponds to the correlation function of coda waves, obtained for an isotropic distribution of sources. 


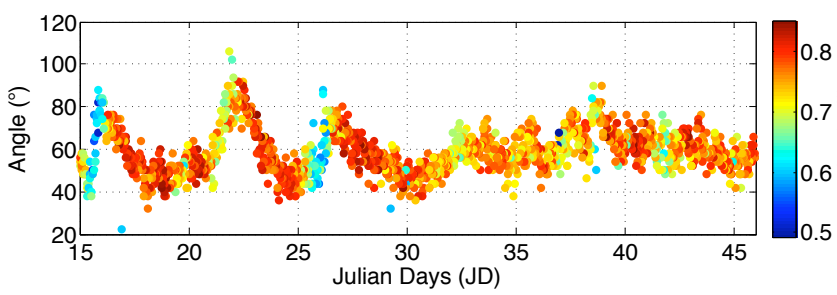

(a)

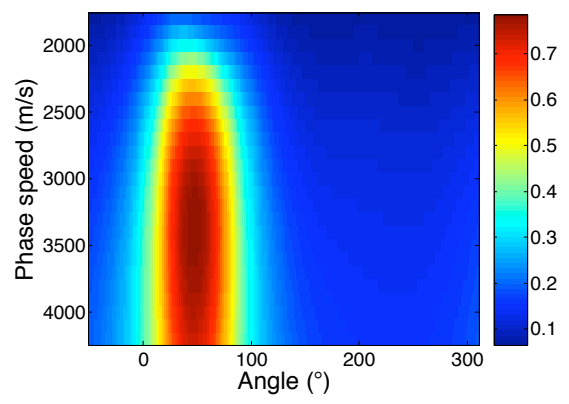

(b)

Figure 1: 


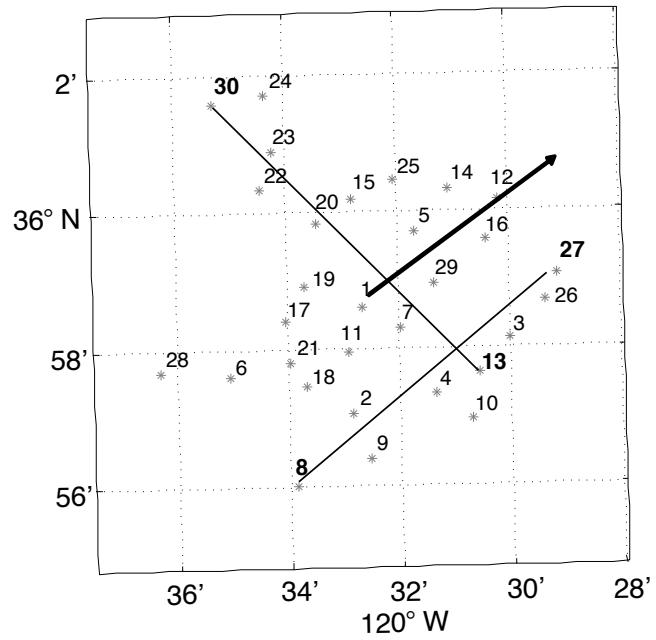

(a)

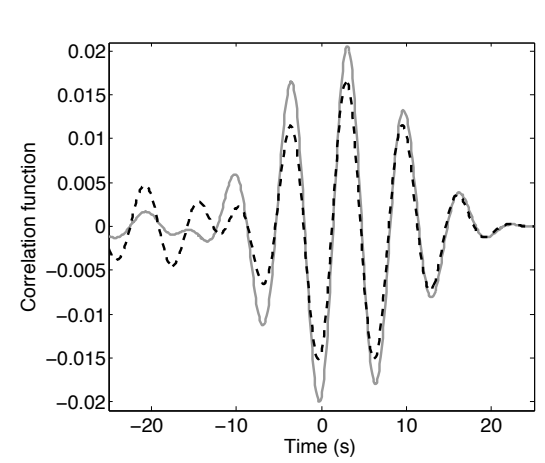

(b) pair 8-27

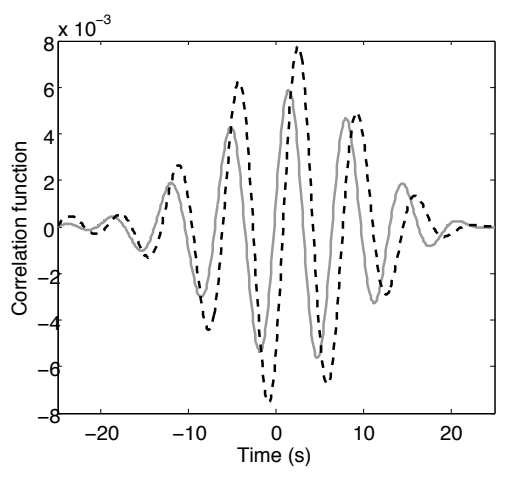

(c) pair $13-30$

Figure 2: 


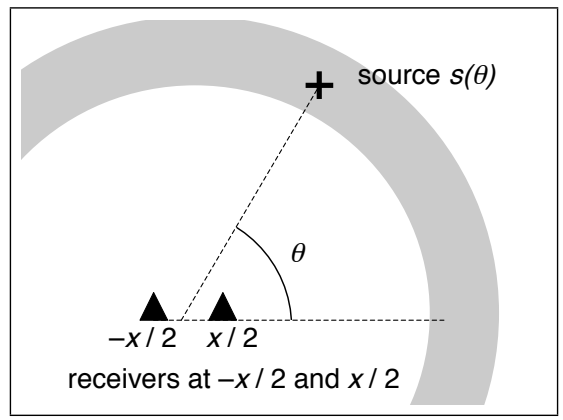

Figure 3:
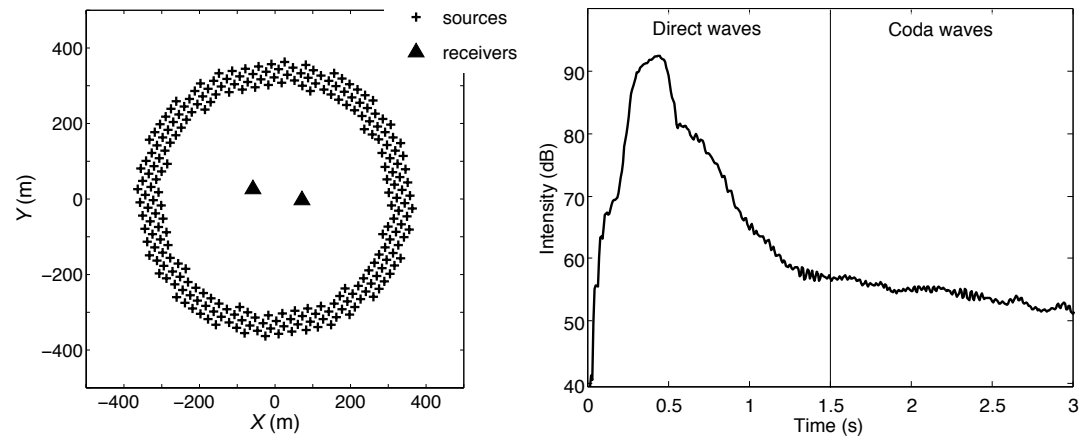

(a)

(b)

Figure 4: 

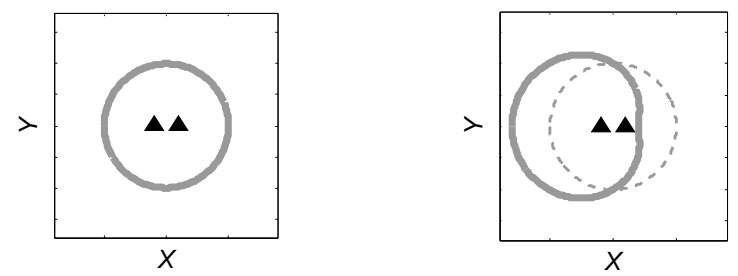

(a)
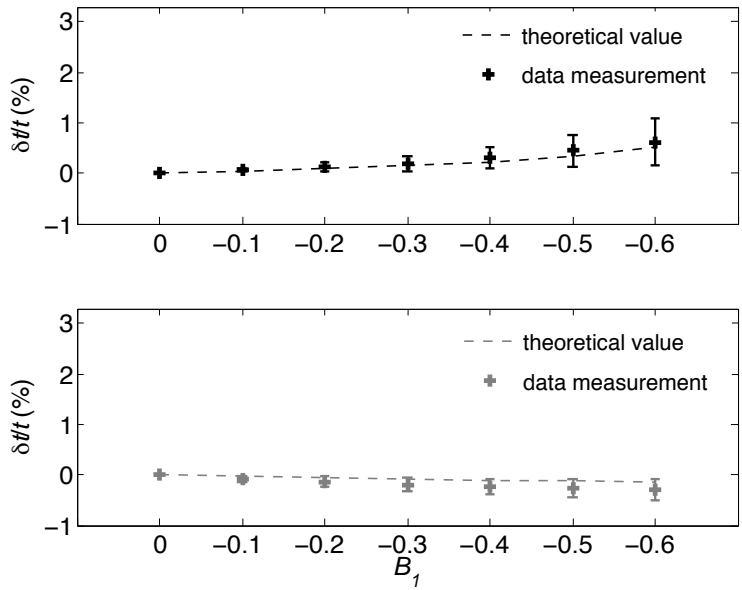

(b)
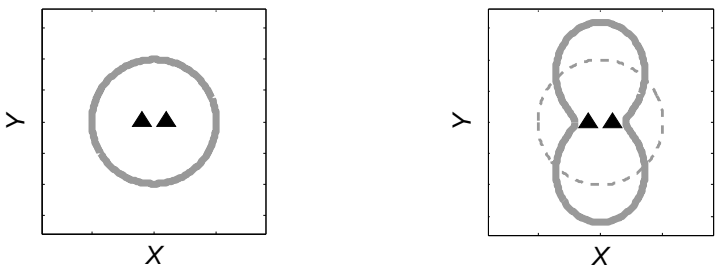

(c)
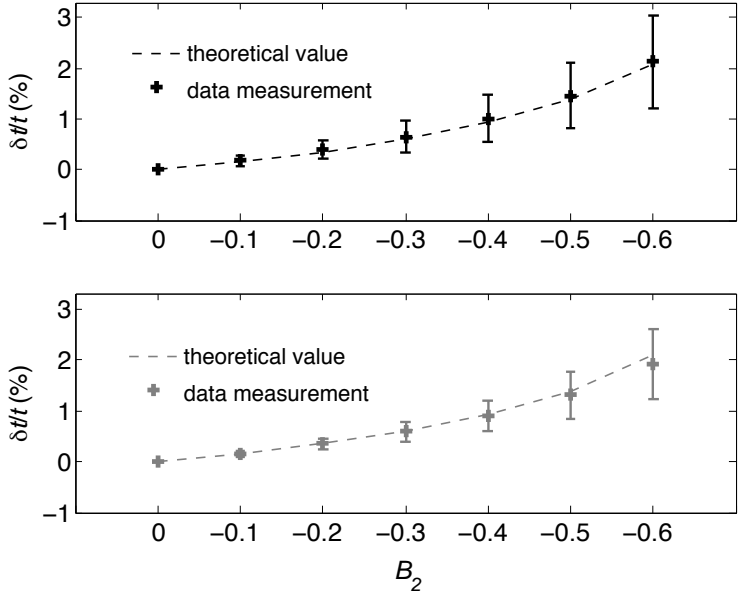

(d)

Figure 5: 


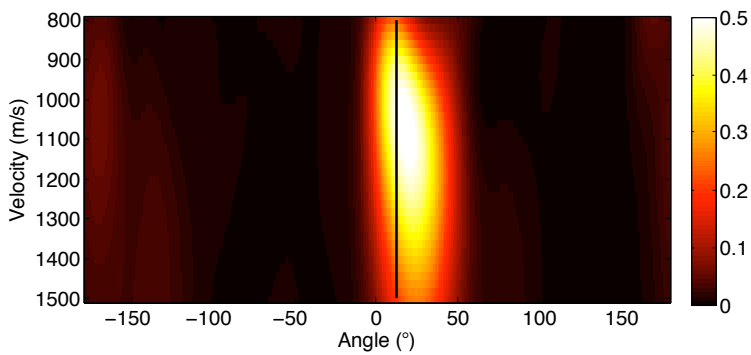

(a)

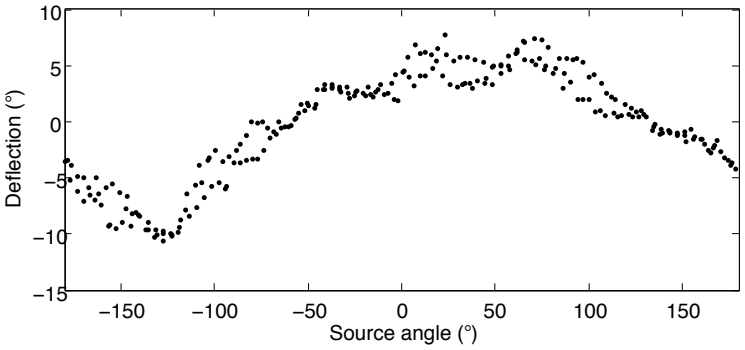

(b)

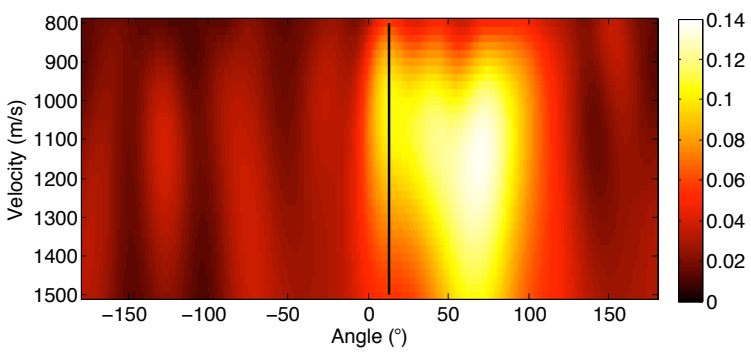

(c)

Figure 6: 


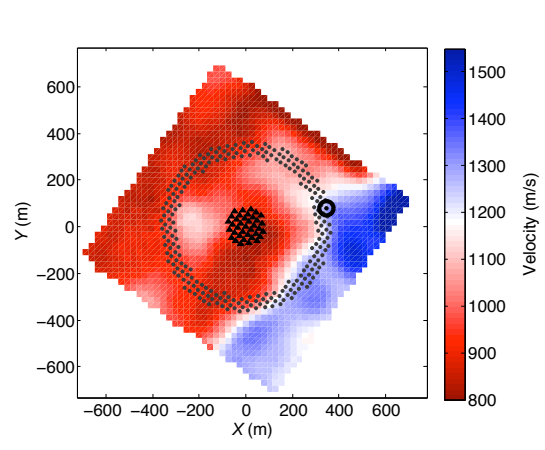

(a)

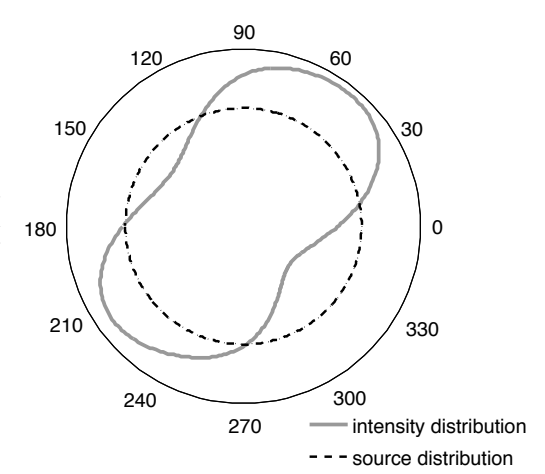

(b)

Figure 7:
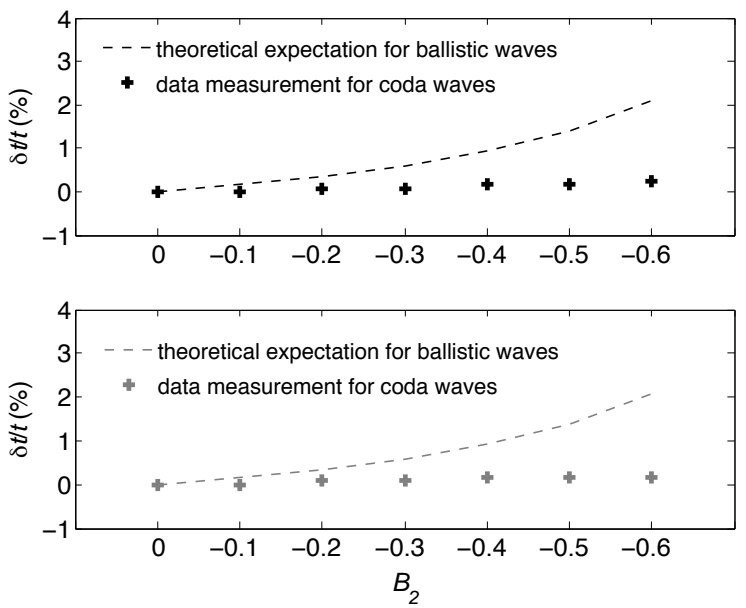

Figure 8: 